\title{
Experimental study of liquid-vapor mass transfer in non-reacting and reacting droplet chains
}

\author{
Michael Stöhr*1, Stefanie Werner ${ }^{1}$, Wolfgang Meier ${ }^{1}$ \\ ${ }^{1}$ German Aerospace Center (DLR), Institute of Combustion Technology, \\ Pfaffenwaldring 38-40, 70569 Stuttgart, Germany \\ *Corresponding author: michael.stoehr@dlr.de
}

\begin{abstract}
The dynamics of liquid-vapor mass transfer largely determines the performance of internal and gas turbine spray combustors. The key mechanisms however typically take place on small spatial scales of less than $100 \mu \mathrm{m}$ which have been difficult to measure. The present work thus aims at the development and application of an experimental technique for the characterization of droplet evaporation with high spatial resolution. Single chains of monodisperse acetone droplets with diameters of 125 and $225 \mu \mathrm{m}$ are injected into a channel with a cross-section of $60 \times 60 \mathrm{~mm}^{2}$ and quartz glass side walls for optical access. The droplet chains are surrounded by a laminar air flow with velocity and temperature of about $0.1 \mathrm{~m} / \mathrm{s}$ and $300 \mathrm{~K}$, respectively. The distribution of acetone vapor around the droplets is measured using planar laser-induced fluorescence (PLIF) excited by the 4th harmonic of a $\mathrm{Nd}$ :YAG laser at $266 \mathrm{~nm}$. The measurements are performed in thin transversal sections between the droplets in order to avoid signal corruption by halation effects that occur when the laser directly hits the droplets as reported in previous studies. In addition, the spatial resolution of the PLIF setup was enhanced by using proper sheetforming and imaging optics. The resulting in-plane resolution and out-plane-resolution (i.e. thickness of the laser sheet) are both determined to about $20 \mu \mathrm{m}$, which thus allows an accurate characterization of the small-scale vapor distribution near the droplets. Using a separate calibration measurement, quantitative acetone concentrations are obtained for non-reacting conditions. As a complementary technique, the droplet evaporation is measured using shadowgraphy droplet sizing. Both non-reacting and reacting droplet chains are studied. The results for the non-reacting cases show that the droplet chains are surrounded by a column of nearly-saturated acetone vapor with a concentration maximum at the centerline. For increasing radial distances, the vapor concentration decays quickly with a half width of $0.5 \mathrm{~mm}$ and reaches almost zero for $r>1 \mathrm{~mm}$. It is further seen that the width of the vapor column increases with streamwise distance. For the experiment with a reacting droplet chain, which is continuously ignited by a heating wire at the channel inlet, a cylindrical reaction zone around the chain with a radius of about $1.5 \mathrm{~mm}$ is observed. The shadowgraphy measurements show that the rate of droplet evaporation is significantly enhanced for the reacting conditions. This is attributed to the high rate of heat transfer from the flame to the droplets and the resulting enhanced acetone mass transfer to the sink at the reaction zone.
\end{abstract}

\section{Keywords}

Droplet evaporation, Laser-induced fluorescence, Monodisperse droplet chain, Liquid-vapor mass transfer

\section{Introduction}

Several important energy conversion devices such as internal combustion engines and gas turbines are driven by the combustion of liquid fuels. The liquid fuels are typically first atomized into a spray of small droplets, which are then dispersed in a turbulent flow, and subsequently evaporate, and then mix and react with air. In many cases the evaporation is the slowest, i.e., rate-controlling process. The detailed experimental characterization of droplet evaporation, especially for an increasing number of alternative fuels, is therefore an important basis for an enhanced understanding of the physical mechanisms and the advancement of numerical models needed for the design of improved combustors.

A classical canonical configuration for the investigation of droplet evaporation and combustion is the linear monodisperse droplet chain. Several quantities have been characterized experimentally for this configuration, such as, e.g., gas phase temperature using coherent anti-stokes Raman spectroscopy (CARS) [1-3], droplet temperature using laser-induced fluorescence (LIF) [4-5] and rainbow thermometry (RT) [6-8], vapor concentration using planar LIF (PLIF) [9, 10], and droplet size using RT, interferometric laser imaging for droplet sizing (ILIDS) [10,11] and droplet imaging $[12,13]$ (mentioning only a part of the numerous works).

The dynamics of the liquid-vapor mass transfer, however, is governed by strong gradients of concentration and temperature in the close vicinity of the gas-liquid interface. Due to the typically small size of droplets, the key mechanisms therefore take place on small spatial scales of significantly less than $100 \mu \mathrm{m}$, which have been 
difficult to measure. The spatial resolution of methods for measurement of vapor concentration near droplets, which are usually performed using PLIF of a fluorescent species such as acetone [9, 10], e.g., was limited by the thickness of the laser sheet of at least $150 \mu \mathrm{m}$, and therefore an improvement is desirable.

The measurement of vapor concentration in the gas phase near the droplet interface is further complicated by socalled halation effects. These effects are caused by interaction of laser light with the fluorescent liquid and lead to corruption of the PLIF signal within a distance of about one droplet diameter from the interface as reported in Refs. $[9,10]$.

The present work thus aims at the development and application of a PLIF method with enhanced spatial resolution that allows the accurate characterization of liquid-vapor mass transfer close to droplet boundaries. For this purpose, the halation effects, which occurred in previous studies using an axial PLIF configuration (Figure 1a) are avoided by a transverse PLIF setup where the horizontal laser sheet is located between two adjacent droplets (Figure 1b). In addition, a significantly enhanced spatial resolution of the PLIF setup was achieved by usage of selected sheet-forming and imaging optics. Using a separate calibration measurement, quantitative acetone concentrations are obtained for non-reacting conditions.

The measuring technique is applied to monodisperse droplet chains operated in a laminar flow channel with welldefined boundary conditions. Both non-reacting and reacting droplet chains are studied and then compared, and the benefits and limitations of the measuring technique are discussed.
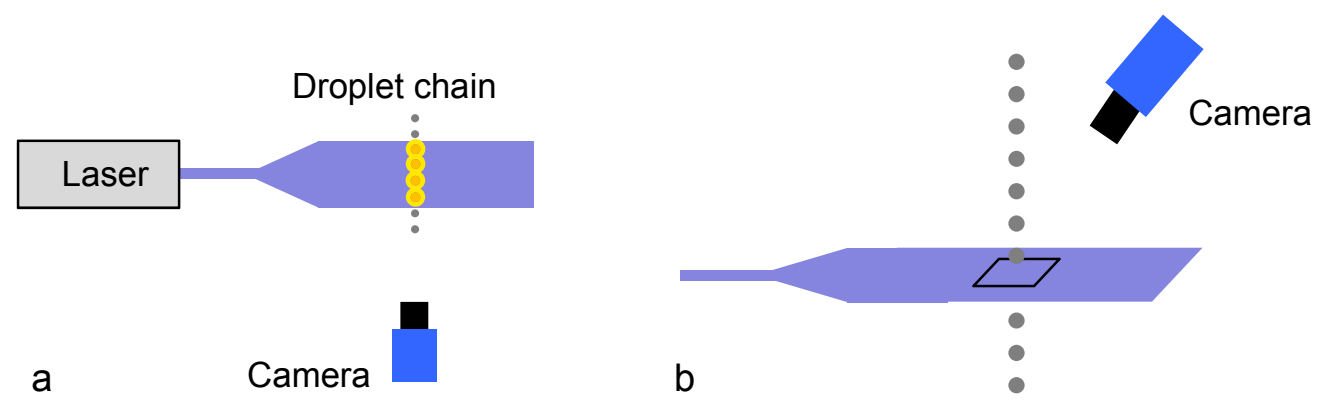

Figure 1: Configurations for (a) axial and (b) transverse vapor PLIF imaging around droplet chains.

\section{Experimental setup}

Measurements are performed in a flow channel (shown in Figure 2) with a quadratic cross-section of $60 \times 60 \mathrm{~mm}^{2}$ and a height of $200 \mathrm{~mm}$. All four side walls consist of glass windows (thickness $8 \mathrm{~mm}$ ) held by corner posts in order to provide optical access. Air is introduced into a plenum at the channel base and then passes through a porous sintered bronze plate into the channel. Air preheating is possible, but not used in the present work. Monodisperse chains of acetone droplets are produced by Rayleigh decay of a laminar liquid jet using a piezoelectric droplet generator (FMP Technology). Orifice plates with diameters of $50 \mu \mathrm{m}$ and $100 \mu \mathrm{m}$ are used, leading to droplet diameters of $125 \mu \mathrm{m}$ and $225 \mu \mathrm{m}$ as described further below. In all cases a laminar air flow with a temperature of about $\mathrm{T}=300 \mathrm{~K}$ and an average velocity of about $0.1 \mathrm{~m} / \mathrm{s}$ is applied. The droplet chains can be operated under both non-reacting and reacting conditions. In the latter case, a Kanthal heating wire wrapped onto a ceramic pipe surrounding the droplet chain at the channel inlet provides a continuous ignition source. The outlet of the ceramic pipe is defined as the origin $y=0 \mathrm{~mm}$ of the axial distance $\mathrm{y}$.

The acetone vapor concentration in the gas phase near the droplets is measured using the vapor fluorescence induced by laser light with a wavelength of $\lambda=266 \mathrm{~nm}$. The output of a frequency-quadrupled Nd:YAG laser (Innolas Spitlight 600, pulse duration ca. $7 \mathrm{~ns}$, operated at a repetition rate of $8 \mathrm{~Hz}$ and a pulse energy of $6 \mathrm{~mJ}$ at $266 \mathrm{~nm}$ ) is formed to a laser sheet using a cylindrical lens of $f=150 \mathrm{~mm}$ focusing on the droplet chain. The fluorescence emission in the range of $350-550 \mathrm{~nm}$ is recorded by two cameras: The first (Camera 1 in Figure 2a, Lavision Imager Intense equipped with a $\mathrm{f}=\mathbf{2 0 0} \mathrm{mm}$ Nikon F/4 macro lens) is located at the same height as the laser sheet, and thus records a 1D profile of the PLIF signal integrated across the laser sheet. The second camera (Camera 3 in Figure 2a, Lavision Imager SCMOS equipped with the same macro lens and mounted on a Scheimpflug adapter) is located laterally above the laser sheet and thus records the 2D PLIF distribution in the plane of the laser sheet. Perspective distorsions due to the diagonal view are corrected in the post-processing 
using an image transformation based on a geometrical calibration. The fields of view (FOV) of the two cameras are specified in Figure 2a.

Droplet sizes are measured using shadowgraphy. The $\lambda=532 \mathrm{~nm}$ output of the PLIF laser is transformed into a diverging beam using a $\mathrm{f}=-20 \mathrm{~mm}$ concave spherical lens, such that the beam illuminates a fluorescent screen with a diameter of about $100 \mathrm{~mm}$. The incoherent red fluorescence of the screen is recorded by a CCD camera (Camera 2 in Figure 2a, Lavision Imager Intense) equipped with a long-distance microscope (Questar QM 100) with a working distance of about $250 \mathrm{~mm}$ and a field of view of $0.97 \times 0.73 \mathrm{~mm}^{2}$.

Both the PLIF and shadowgraphy measurements are synchronized to the droplet formation by using the signal that drives the piezo-electric droplet generator as an external trigger for laser and cameras.
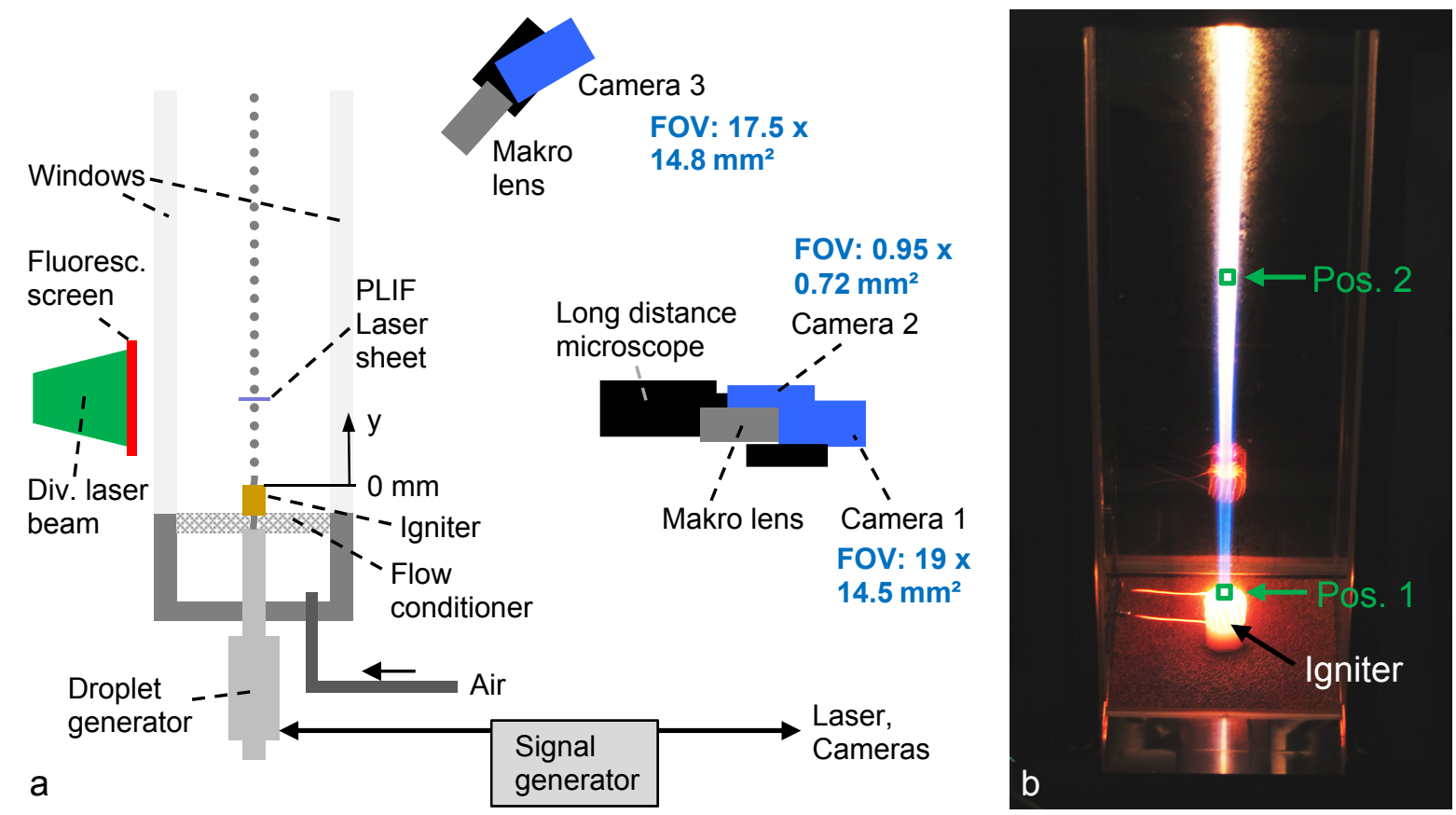

Figure 2: (a) Sketch of experimental setup and (b) photograph of reacting droplet chain in the flow channel.

\section{Characterization of spatial resolution}

In order to measure vapor distributions in the gas phase between droplets with diameters in the range of 100$250 \mu \mathrm{m}$, a spatial resolution of significantly less than $100 \mu \mathrm{m}$ is desirable. For planar measurement techniques such as PLIF, spatial resolution generally has two aspects: The 2D in-plane resolution that is governed by the imaging optics, and the out-of-plane resolution that is determined by the thickness of the laser sheet. The 2D inplane resolution was measured by imaging the line pattern of a USAF 1951 target as shown in Figure 3 . With the standard windows of $8 \mathrm{~mm}$ thickness it was found that the astigmatism due to the diagonal view through the glass limits the resolution to about $150 \mu \mathrm{m}$. As a consequence, the side window towards the PLIF cameras was replaced by a $1 \mathrm{~mm}$ glass plate mounted onto a metal frame. The central part of the resulting target image shown in Figure $3 b$ shows that the resolution is then significantly enhanced such that lines of at least $20 \mu \mathrm{m}$ can be resolved.

The thickness of the laser sheet in the vicinity of the droplet chain is characterized using a beamprofiler (Gentec Beamage $4 \mathrm{M}$ ) that is traversed across the focus of the sheet-forming cylindrical lens as shown in Figure 4a. The results plotted in Figure $4 \mathrm{~b}$ show that the thickness is in the range 15-20 $\mu \mathrm{m}$ within a distance of $\pm 0.5 \mathrm{~mm}$ around the focus. Since the acetone vapor is mostly confined to a radius of also $0.5 \mathrm{~mm}$ around the droplet chain as shown below, an out-of-plane resolution of $\leq 20 \mu \mathrm{m}$ can be assumed. This fits well to the similar value of $20 \mu \mathrm{m}$ for the in-plane resolution, resulting in an overall 3D resolution of about $20 \mu \mathrm{m}$.

The resolution of the shadowgraphy imaging system using the long-distance microscope is about $2 \mu \mathrm{m}$. 
a
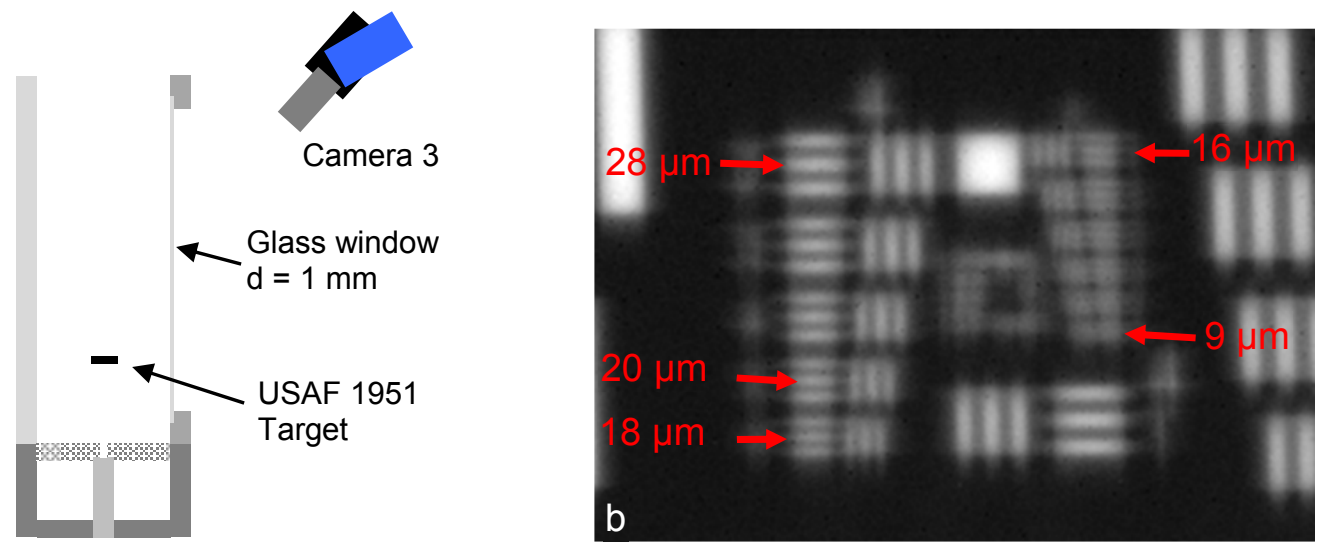

Figure 3: (a) Setup für determination of in-plane spatial resolution, (b) image of USAF 1951 target.

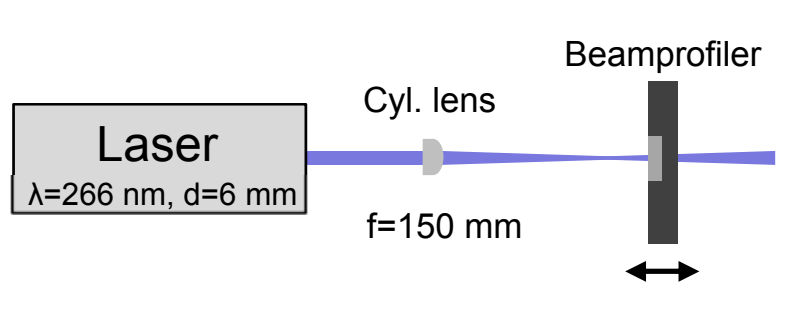

a

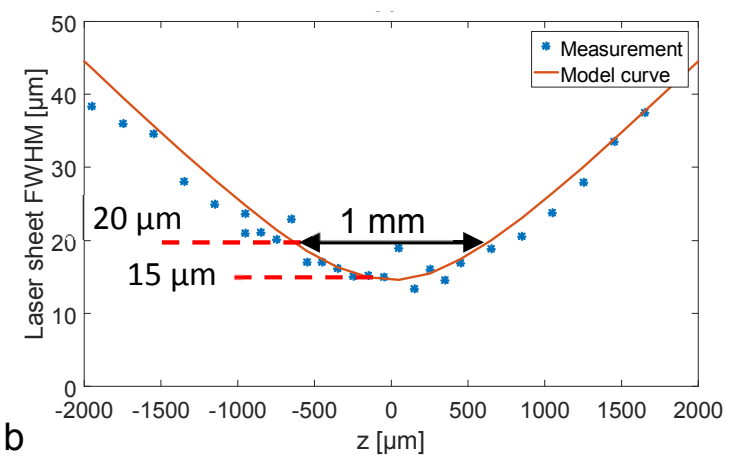

Figure 4: (a) Setup for measurement of laser sheet thickness, (b) sheet thickness near focus $(z=0 \mu m)$.

\section{Calibration of PLIF signal}

In order to determine quantitative acetone vapor concentrations from the $2 \mathrm{D}$ acetone PLIF signals measured with camera 3, a separate calibration measurement is performed. A stream of saturated acetone at a temperature of $300 \mathrm{~K}$ is introduced into the channel directly below the PLIF laser sheet through a small pipe as shown in Figure 5. For this air/vapor mixture, which has a known acetone concentration of $0.013 \mathrm{~mol} / /$, the PLIF signal is then recorded with same laser and camera settings as in the droplet chain measurements.

The resulting PLIF image is shown in the right part of Figure 5. Intensity variations in the vertical direction are caused by inhomogeneities of the laser beam. In the horizontal direction, an intensity minimum is observed in the central part where the focus is located. This intensity reduction is caused by saturation of the fluorescence due to excessive fluence of the laser pulse, which is highest at the focus. A further increase of laser pulse energy therefore would not lead to an increased PLIF signal.

The droplet PLIF measurements are then corrected for the effects from laser beam inhomogeneity and the fluorescence saturation through a division by the calibration measurement. The resulting data then provides a $2 \mathrm{D}$ distribution of volumetric acetone concentration in mol/l.

It is noted that the acetone fluorescence quantum yield generally depends on temperature [14]. For an excitation at $\lambda=266 \mathrm{~nm}$, however, the variation of fluorescence per molecule is less than $5 \%$ per $100 \mathrm{~K}$ for temperatures around $300 \mathrm{~K}$ that are relevant for the present non-reacting conditions [14], and therefore uncertainties due to the variation of fluorescence quantum yield are very low. It is further noted that effects from collisional quenching on acetone fluorescence are negligible [15]. 


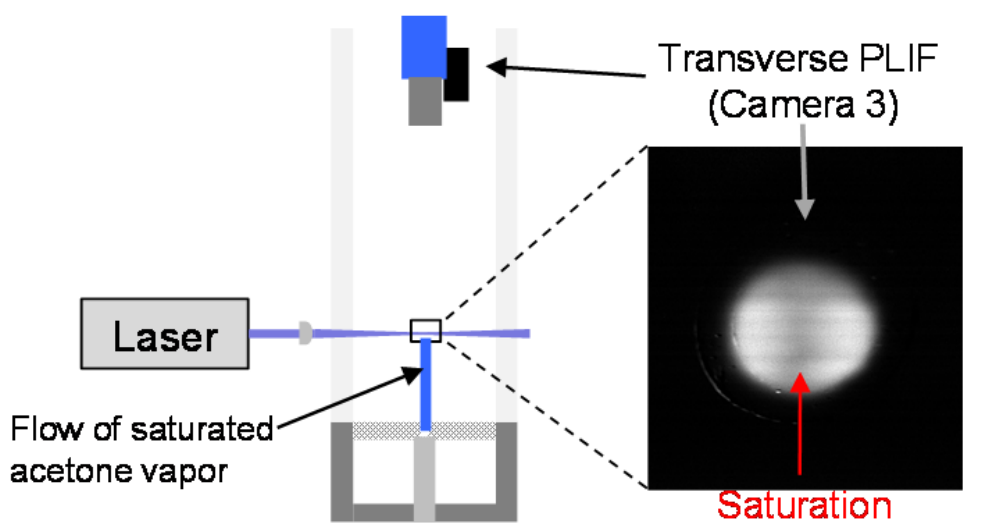

Figure 5: Setup for PLIF signal calibration.

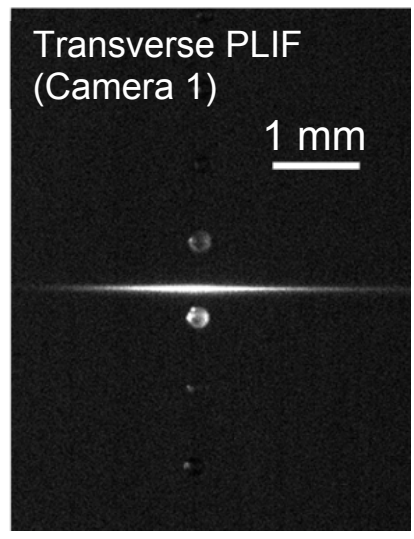

Figure 6: Transverse 1D PLIF distribution for case $A$ at $y=50 \mathrm{~mm}$.

\section{Results for non-reacting condition}

In the following sections, measurements for two droplet chain parameters are presented. In the first case $A$, an orifice plate diameter $d_{0}=100 \mu \mathrm{m}$, droplet diameter $d_{d} \approx 225 \mu \mathrm{m}$, droplet spacing $C \approx 3.9 d_{d}$, and droplet velocity of $v \approx 15 \mathrm{~m} / \mathrm{s}$ were chosen. In the second case $B$, the parameters were $d_{o}=50 \mu \mathrm{m}, d_{d} \approx 125 \mu \mathrm{m}, C \approx 4.1 d_{d}$ and $v \approx 19$ $\mathrm{m} / \mathrm{s}$. For case $B$, measurements were also performed under reacting condition, and are discussed in the next section.

In Figs. 6 and 7, a PLIF measurement for case $A$ at a height of $y=50 \mathrm{~mm}$ in the channel is shown. The 1D horizontal, line-of-sight integrated profile obtained with camera 1 (Figure 6) clearly shows that the laser sheet passes through droplet chain axis without impinging on a droplet. Some droplets near the laser sheet are visible due to laser light reflected at the channel window. Although no quantitative concentrations can be obtained from the line-of-sight integrated 1D profile, the measurement reveals the position of the laser sheet (in this case, about $230 \mu \mathrm{m}$ above the upper border of the droplet below), and indicates that the droplet chain is surrounded by a column of acetone vapor with a radius of roughly $1 \mathrm{~mm}$.

The corresponding 2D PLIF distribution obtained with camera 3 is shown in Figure 7a. Here, 100 instantaneous measurements with the same position relative to the droplets were averaged. The PLIF image shows a continuous distribution of acetone vapor with up to 20 counts as well as superimposed signals from the droplets below and above the laser sheet that are illuminated by reflected laser light as mentioned above. In the region with superimposed droplets, apparently no vapor concentrations can be estimated. The resulting quantitative distribution of vapor concentration, obtained with the calibration described in the previous section, is shown in Figure $7 \mathrm{~b}$. From the 2D distribution, a $1 \mathrm{D}$ profile through the centerline is plotted in Figure 7c. The profile shows that the vapor concentration reaches a maximum at the centerline of about $60 \%$ of the saturation concentration at $\mathrm{T}=300 \mathrm{~K}$, i.e. about $7.810^{-3} \mathrm{~mol} / \mathrm{l}$, and has a half width of about $0.5 \mathrm{~mm}$. The concentration distribution roughly resembles a Gaussian, which would be consistent with a classical diffusion-like radial mass transport.

From the measurements for case B, horizontal line-of-sight integrated PLIF profiles and shadowgraphy images obtained at heights of $y=3 \mathrm{~mm}$ and $\mathrm{y}=93 \mathrm{~mm}$ (labeled as Pos.1 and 2, respectively, in Figure 2b) are shown in Figure 8. While at $y=3 \mathrm{~mm}$ the column of acetone vapor around the droplets has a width of about $2 \mathrm{~mm}$, further downstream at $y=93 \mathrm{~mm}$ the width is increased to about $4 \mathrm{~mm}$. This demonstrates the radial transport of acetone vapor in the laminar channel flow. A similar growth of vapor column width for increasing distance from the injector has also been observed in a recent numerical simulation of a monodisperse droplet chain [16]. From the corresponding shadowgraphy images, the droplet size is estimated as $\approx 125 \mu \mathrm{m}$ for both positions, i.e. no significant decrease of droplet size appears. This is expectable since the droplets move through a near-saturated acetone vapor, and the heat transfer from the ambient air at $\mathrm{T} \approx 300 \mathrm{~K}$ is low. 


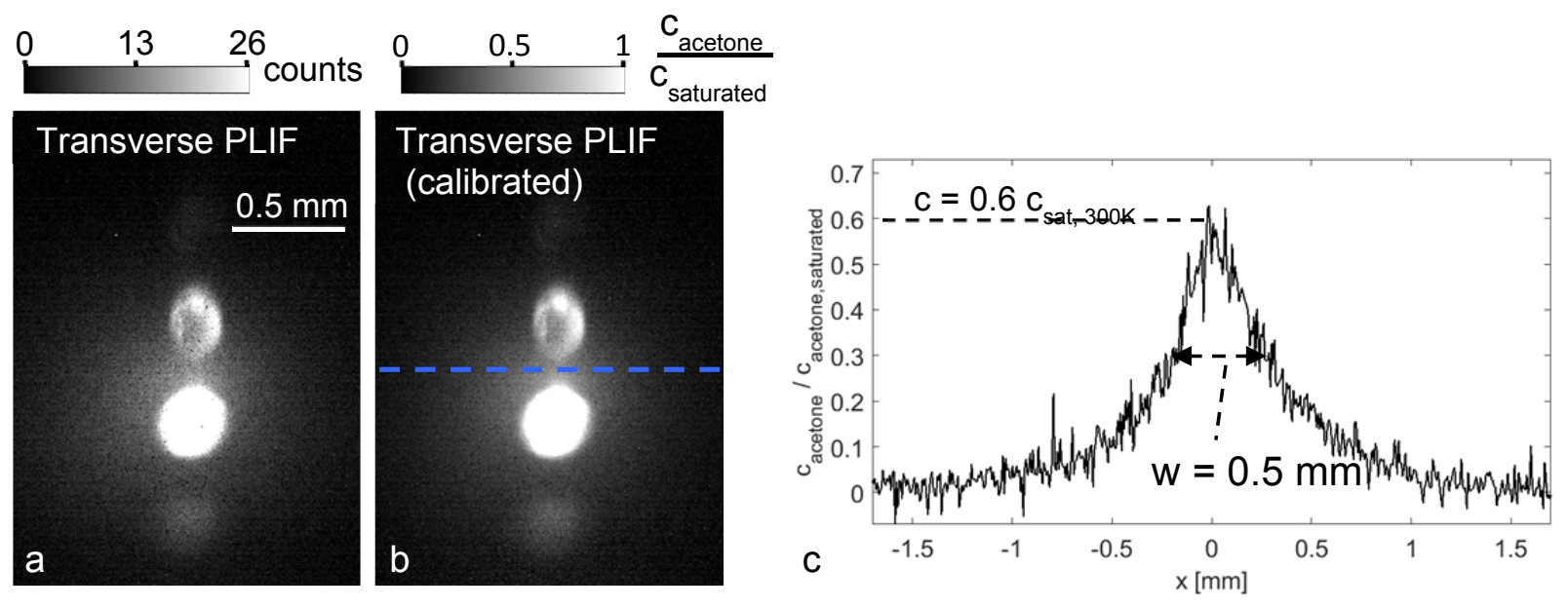

Figure 7: (a) Raw and (b) calibrated transverse distributions of PLIF signal for case $A$ at $y=50 \mathrm{~mm}$, (c) concentration profile along a line (marked blue in (b)) across the droplet chain axis.

\section{Results for reacting condition}

The droplet evaporation and liquid-vapor mass transfer for the reacting case B differ significantly from those of the non-reacting cases discussed above. When the fuel vapor is continuously ignited by the heating wire at the channel base, a cylindrical flame encloses the droplet chain as shown in Figure $2 \mathrm{~b}$. Chemiluminescence images (not shown) indicate that the flame front is located at a radius of about $r=1.5 \mathrm{~mm}$. It is further observed that for heights of $y>50 \mathrm{~mm}$, significant levels of soot are formed in the flame.

The horizontal PLIF profiles at $y=3 \mathrm{~mm}$ displayed in Figure 8 show that the PLIF intensities are significantly lower for the reacting condition than for the corresponding non-reacting case. This is expected for two reasons: First, CARS measurements for reacting monodisperse droplet chains showed that gas temperatures on the centerline are typically in the range of 1200-2000 K [1-3], which implies a strongly reduced gas density and thereby a reduced volumetric vapor concentration. Secondly, the fluorescence quantum yield of acetone for excitation at $\lambda=266 \mathrm{~nm}$ is significantly reduced for temperatures larger than, say, $600 \mathrm{~K} \mathrm{[14]}$. Since the gas temperatures for the present experiment are unknown, these two effects cannot be corrected and therefore no quantitative vapor concentrations can be obtained for the reacting case.

At the height $\mathrm{y}=93 \mathrm{~mm}$, by contrast, a much stronger PLIF signal appears for the reacting condition compared to the non-reacting case. The photograph in Figure $2 \mathrm{~b}$ indicates that intense soot formation takes place in this part of the flame. The region of soot formation can further be identified as a gray vertical column caused by soot luminosity in the PLIF image for $y=93 \mathrm{~mm}$ in Figure 8. The high PLIF signal is therefore attributed to PAH molecules, which are considered as soot precursors and strongly fluoresce when excited at $\lambda=266 \mathrm{~nm}$ [17]. While the PAH fluorescence further inhibits the quantification of acetone vapor in the sooting regions of the reacting droplet chain, it may enable insights into the onset of soot formation.

The corresponding shadowgraphy images show that the droplet diameters have reduced considerably from 125 $\mu \mathrm{m}$ at the channel base to $112 \mu \mathrm{m}$ at $\mathrm{y}=93 \mathrm{~mm}$. This is apparently caused by the intense heat transfer from the flame front at $r=1.5 \mathrm{~mm}$ to the droplets at $r=0 \mathrm{~mm}$. As a rough estimate, the CARS measurements for a reacting chain of monodisperse ethanol droplets by Virepinte et al. [3] showed that the gas temperature reaches a maximum of $2000 \mathrm{~K}$ at the flame front at $\mathrm{r}=1.5 \mathrm{~mm}$ and reduces to $1300-1500 \mathrm{~K}$ at the centerline, which corresponds to a substantial temperature gradient of $330-470 \mathrm{~K}$ per $\mathrm{mm}$ driving the fuel vaporization. 


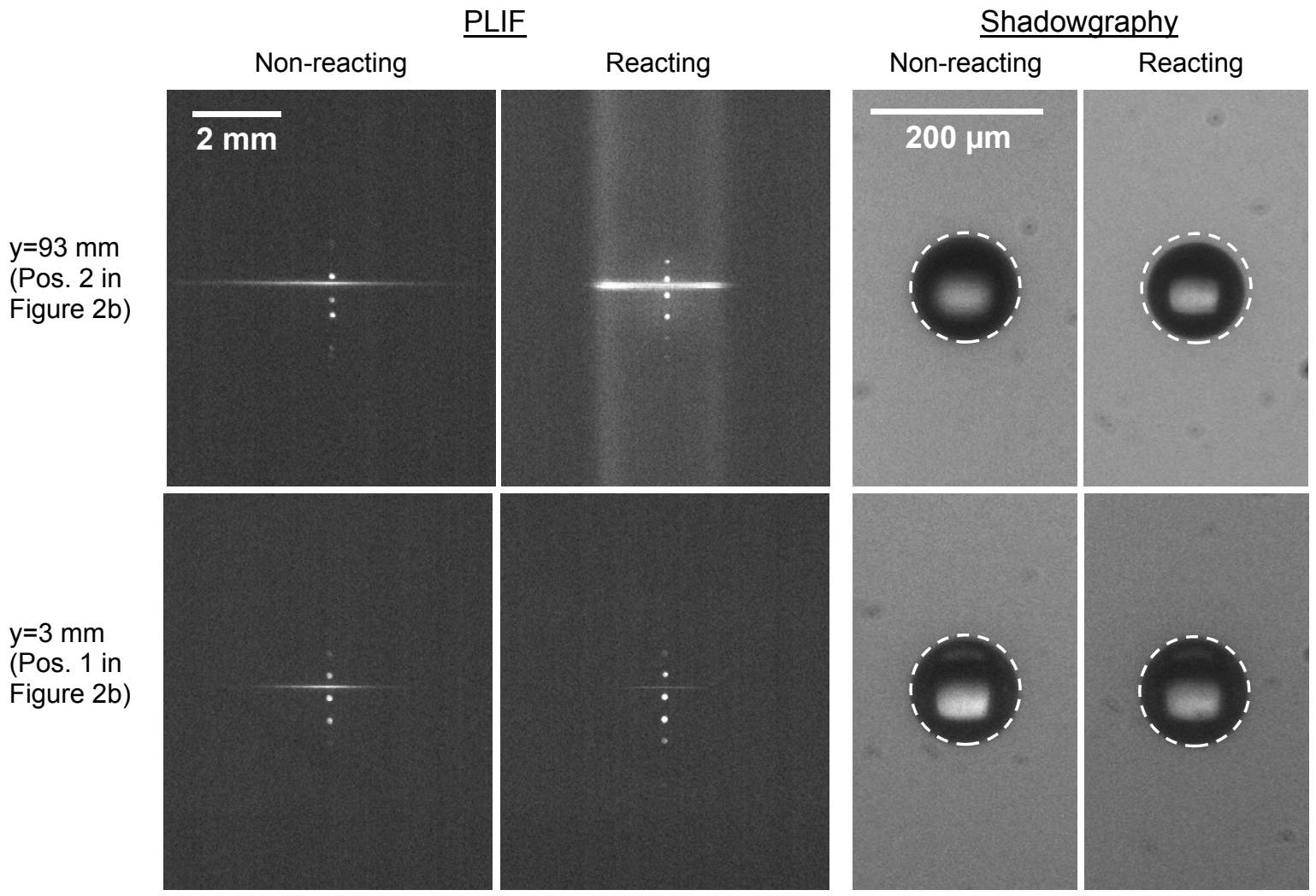

Figure 8: Transverse vapor PLIF (left) and droplet shadowgraphy (right) for non-reacting and reacting conditions for case $B$ at heights $y=3 \mathrm{~mm}$ and $y=93 \mathrm{~mm}$. The dashed circles represent a diameter of $125 \mu \mathrm{m}$.

\section{Conclusions}

The present work demonstrated the development and application of a PLIF technique for the characterization of the fuel vapor distribution around monodisperse chains of acetone droplets in a laminar flow channel. The technique features a high spatial resolution of around $20 \mu \mathrm{m}$, which is significantly improved compared to prior approaches. Furthermore the signal corruption by halation effects due to interaction of laser light and droplets, which was reported in previous studies, could be avoided by measurements in thin transversal sections between the droplets. Together with a calibration using the signal from air with known acetone vapor concentration, quantitative acetone concentrations could be obtained for the first time, to our knowledge, in the close vicinity of droplets.

Measurements were performed for two cases denoted as A and B with different droplet diameters and spacings. The latter case $B$ was also studied under reacting conditions using a heating wire as a continuous ignition source. The results for the non-reacting case A revealed a cylindrical column of acetone vapor around the droplet chain with a Gaussian-like concentration distribution that reaches a maximum value of $7.810^{-3} \mathrm{~mol} / \mathrm{l}$ near the centerline at a height of $y=50 \mathrm{~mm}$. The results for the non-reacting case $B$ showed that the radius of the column of nearsaturated acetone vapor increases in streamwise direction from $r \approx 1 \mathrm{~mm}$ at the channel base to $r \approx 2 \mathrm{~mm}$ at a height of $y=93 \mathrm{~mm}$. The evaporation rate of the non-reacting droplets determined using shadowgraphy imaging was found to be very low, as expected from the low heat transfer and the nearly-saturated vapor in the vicinity of the droplets.

For the corresponding reacting case $\mathrm{B}$, the droplet chain is enclosed by a cylindrical flame front with a radius of about $r=1.5 \mathrm{~mm}$. The acetone PLIF signal around the droplets is significantly reduced compared to the nonreacting case as expected from the reduced gas density and reduced fluorescence yield at higher gas temperature. For heights of $y>50 \mathrm{~mm}$, significant levels of soot are formed in the flame. Due to the elevated, yet not precisely known temperatures, and additional interference with PAH fluorescence in the soot-forming regions, acetone vapor concentrations could not be quantified for the reacting case. Shadowgraphy measurements of droplet size, on the other hand, indicated a significantly enhanced rate of vaporization for the reacting case, which is consistent with the strong heat transfer from the flame front. 


\section{Acknowledgements}

The contribution of Tobias Biesner to the design and setup of the experiment is appreciated. Financial support from the DLR project R2F is gratefully acknowledged.

\section{References}

[1] Zhu, J., Dunn-Rankin, D., Applied Optics, 1991, 30, pp. 2672-2674.

[2] Zhu, J., Dunn-Rankin, D., 1992, Symposium (International) on Combustion, 24, pp. 1473-1481.

[3] J.F. Virepinte, Y. Biscos, G. Lavergne, P. Magre, G. Collin, 2000, Combustion Science and Technology, 150, pp. 143-159.

[4] Castanet, G., Lemoine, F., 2007, Proceedings of the Combustion Institute, 31, pp. 2141-2148.

[5] Lemoine, F., Castanet, G., 2013, Experiments in Fluids, 54, 1572.

[6] Roth, N., Anders, K., Frohn, A., 1991, Applied Optics, 30, pp. 4960-4965.

[7] Castanet, G., Lavielle, P., Lemoine, F., Lebouche, M., Atthasit, A., Biscos, Y, Lavergne, G., 2002, International Journal of Heat and Mass Transfer, 45, pp. 5053-5067.

[8] Saengkaew, S., Godard, G., Blaisot, J. B., Grehan, G., 2009, Experiments in Fluids, 47, pp. 839-848.

[9] Orain, M., Mercier, X., Grisch, F., 2006, Combustion Science and Technology 177, pp. 249-278.

[10] Sahu, S., Hardalupas, Y., Taylor, A. M. K. P., 2014, Experiments in Fluids, 55, 1673.

[11] Glover, A. R., Skippon, S. M., Boyle R. D., 1995, Applied Optics, 34, pp. 8409-8421.

[12] Silverman, M. A., Dunn-Rankin, D., 1994, Combustion Science and Technology, 100, pp. 57-73.

[13] Perrin, L., Castanet, G., Lemoine, F., 2015, Experiments in Fluids, 56, 29.

[14] Thurber, M. C., Grisch, F., Kirby, B. J., Votsmeier, M., Hanson, R.K., 1998, Applied Optics, 37, pp. 49634978.

[15] Lozano, A., Yip, B., Hanson, R. K., 1992, Experiments in Fluids, 13, pp. 369-376.

[16] Castanet, G., Perrin, L., Caballina, O., Lemoine, F., 2016, International Journal of Heat and Mass Transfer, 93, pp. 788-802.

[17] Sun, R., Zobel, N., Neubauer, Y., Chavez, C. C., Behrendt, F., 2010, Optics and Lasers in Engineering, 48, pp. 1231-1237. 